\title{
The Electronic Numerical Integrator and Computer (ENIAC)
}

1. Introduction. It is our purpose in the succeeding pages to give a brief description of the ENIAC and an indication of the kinds of problems for which it can be used. This general purpose electronic computing machine was recently made public by the Army Ordnance Department for which it was developed by the Moore School of Electrical Engineering. The machine was developed primarily for the purpose of calculating firing tables for the armed forces. Its design is, however, sufficiently general to permit the solution of a large class of numerical problems which could hardly be attempted by more conventional computing tools.

In order easily to obtain sufficient accuracy for scientific computations, the ENIAC was designed as a digital device. The equipment normally handles signed 10-digit numbers expressed in the decimal system. It is, however, so constructed that operations with as many as 20 digits are possible.

The machine is automatically sequenced in the sense that all instructions needed to carry out a computation are given to it before the computation commences. It will be seen below how these instructions are given to the machine.

2. Description of the Machine as a Whole. The machine is a large $U$ shaped assemblage of 40 panels (see Figs. 1 and 2) which together contain approximately 18,000 vacuum tubes and 1500 relays. These panels are grouped to form 30 units (see Fig. 2), each of which performs one or more of the functions requisite to an automatic computing machine.

The units concerned mainly with arithmetic operations are 20 accumulators (for addition and subtraction), a multiplier, and a combination divider and square rooter.

Numbers are introduced into the ENIAC by means of a unit called the constant transmitter which operates in conjunction with an IBM card reader. The reader scans standard punched cards (which hold up to 80 digits and 16 signs) and causes data from them to be stored in relays located in the constant transmitter. The constant transmitter makes these numbers available in the form of electrical signals as they are required. Similarly, results computed in the ENIAC may be punched on cards by the ENIAC's printer unit operating in conjunction with an IBM card punch. Tables can be automatically printed from the cards by means of an IBM tabulator.

The numerical memory requirements of the machine are met in several ways. Three function table units provide memory for tabular data. Each function table has associated with it a portable function matrix with switches on which can be set 12 digits and 2 signs for each of 104 values of an independent variable. While primarily designed for the storage of tabular functions, the function table memory can be utilized for any numbers known before a computation begins. Switches for 20 digits and 4 signs on the constant transmitter can also be used for such numbers. Numbers formed in 
the course of a computation and needed in subsequent parts of a computation can be stored in accumulators. Should the quantity of numbers formed during a computation and needed at a later time exceed the accumulator storage capacity, these numbers can be punched on cards and, later, can be reintroduced by means of the card reader and constant transmitter.

The ENIAC's programming memory (memory for instructions relevant to a particular computation) is chiefly local in nature, i.e. instructions are given to each unit. Sets of switches called program switches are located on the front faces of the various units. Before a computation begins these switches are set to specify which particular operations in a unit's repertoire are to be performed. Discussion of the manner in which the temporal order of operations (sequencing) is established will be postponed until section 7 . A unit called the master programmer unit provides a certain amount of centralized programming memory. This topic, too, will be discussed at greater length in section 7.

The remaining two units, the initiating and cycling units, govern the operation of the others. The initiating unit has controls for turning the power on and off, initiating a computation, clearing the machine, and incorporates certain testing features. The cycling unit supplies the fundamental signals upon which the various units operate, and synchronizes the machine.

3. Synchronization. Every 1/5000th of a second the cycling unit emits a fundamental pattern of signals, nine trains of special pulses and a gate. The terms pulse and gate both refer to a change in voltage, either positive or negative, from some reference level. The distinction between these is their duration. In the ENIAC, pulses last for about 2 micro-seconds; gates, for 10 or more micro-seconds. The individual pulses of the various trains are spaced at 10 micro-second intervals.

One of the pulses emitted by the cycling unit, the central programming pulse (CPP), is of particular importance in this discussion. This pulse, emitted once every 1/5000th of a second, marks the beginning and end of a cycle. When a unit completes an operation, it transmits one of these as a program output pulse. Such a pulse, delivered to another unit, stimulates it to perform a given operation. The other signals supplied by the cycling unit are used in effecting this operation. For example, one of the trains of cycling unit pulses (the 9P) consists of 9 individual pulses, another train consists of only 1 pulse (the $1^{\prime} \mathrm{P}$ ). When an accumulator is called upon to transmit the number it stores, the accumulator does so by emitting appropriate numbers of the $9 \mathrm{P}$ and the $1^{\prime} \mathrm{P}$ which originate in the cycling unit.

All the units have been so designed that they require an integral number of cycles to complete their operations. An accumulator, for example, requires $1 / 5000$ th of a second for an addition or subtraction. For this reason, 1 cycle or $1 / 5000$ th of a second is referred to as an addition time. The operation times for other numerical units of the ENIAC are summarized in the table below.

A number of separate trunk lines have been provided for the transmission of signals representing numbers (see section 6). For this reason and because the units operate in synchronism with one another, it is possible for a number of units to operate simultaneously. This, combined with the fact 
OPERATION TIMES FOR ENIAC UNITS

UNIT

Accumulator

Multiplier

Divider and

Square Rooter

Function Table

Constant

Transmitter
Operation

Receives a number and adds it to number stored in accumulator

or transmits the number or the negative of the number stored in it

$r$ times in succession where $1 \leqslant r \leqslant 9$.

Finds the signed product of a multiplicand with as many as 10 digits by a mulplier, $p$ digits (where $2 \leqslant p \leqslant 10$ ).

Finds $p$ digits of the quotient or of twice the square root with the sign of the former.

Selects the functional value corresponding to a given 2-digit argument or any one of 4 neighboring values, and transmits it, or its negative, $r$ times in succession.

Emits a signed 5- or 10-digit number, previously read from a punched card or stored on its switches.
No. OF ADDition Times 1 ADD. TME $=$ sob. SEC.

$\boldsymbol{r}$

$$
p+4
$$

Approximately $13[p+1]$ (depends on the digits of the arguments).

$$
r+4
$$

1

that the ENIAC depends for its operation not on the slow movement of mechanical parts, but instead on electronic circuits which operate on pulses spaced at 10 micro-second intervals, makes possible the high computing speed of this machine.

Although they do not take an absolutely definite number of addition times to complete operations, the electromechanical devices which operate with the ENIAC, namely the IBM card reader and card punch, have also been integrated into the synchronized system by providing for the emission of a programming pulse when one of these devices finishes reading or punching. It might be remarked that these devices operate at much lower speed than the ENIAC. Card reading requires approximately 0.5 seconds; punching about 0.6 seconds. To take advantage of the ENIAC's potentialities, it is desirable for computations or for card punching to proceed in parallel with card reading. There is no danger of initiating another set of operations until both the reading and its parallel operations have been completed since the reader does not emit a program output pulse until it has interlocked with the rest of the ENIAC by receiving a program pulse indicating completion of the parallel operations.

4. Electronic Elements and their Influence on Logical Characteristics. The circuits of the ENIAC are designed around a relatively small number of basic electronic elements. In the succeeding paragraphs we shall remark on a few of these elements which importantly influence the logical characteristics of the computer.

The simplest tube used is the triode, so called because it has three characteristic elements: the cathode (surface which gives off electrons), the plate (surface which receives electrons), and the grid (which controls the current passing through the tube). In addition, there is a heater to bring the cathode to the temperature required for it to emit electrons. Other tubes used in the ENIAC are multi-grid tubes, for example, the pentode which has 3 grids. 
In all cases, vacuum tubes in the ENIAC circuits are used only as "onoff" devices instead of as amplitude sensitive devices, i.e. the presence or absence of a signal depends on whether a tube is conducting or not conducting, and not on any measured magnitude of current or voltage. To say that a tube is "on" or conducting means that with the usual convention of sign, current is flowing from the plate to the cathode. This implies that the plate is at a slightly higher voltage than the cathode, but that this voltage drop is small compared to the drop when the tube is turned "off" or nonconducting. Thus, if a tube is turned off, the voltage of the plate is raised and that of the cathode lowered. Hence, when a tube is turned off, the plate emits a positive signal, and the cathode one that is negative. If the tube is turned on, these signs are reversed. Within appropriate limits, a tube is conducting if its grid (or grids) is (or are) kept above a certain voltage, nonconducting if below that voltage. Thus, a tube is turned on by applying a positive signal to its grid (or grids), turned off by a negative signal.

The basic electronic memory device of the ENIAC is the flip-flop or trigger. A flip-flop consists essentially of a pair of triodes so connected that at any given time one, and only one, of the pair can be conducting. When a certain one of the tubes is conducting, the flip-flop is said to be in the normal state; when the other tube is conducting, the flip-flop is in the abnormal state. A flip-flop has two inputs and two outputs. A pulse received on one input (the set input) throws the flip-flop into the abnormal state, in which state it remains until restored to the normal state by a pulse received at its second (or reset) input. When the flip-flop is in the normal state, one output is positive and the other negative. In the abnormal state, the polarity of the outputs is reversed.

Corresponding to each flip-flop in the ENIAC, there is a neon lamp visible on the front of the machine. The neon is so connected to its corresponding flip-flop that it is lit when the flip-flop is in the abnormal state. These neons provide one of the most important visual checks on the operation of the computer since, as will be seen below, they provide an almost complete picture of the numbers in the machine and the operations being performed. In the normal method of operation, when the cycling unit emits its pulses at the 100 kilocycle rate, the neons blink on and off too rapidly for observation. However, a switch on the cycling unit enables the operator to switch the ENIAC into "one addition time" operation in which the fundamental signals for one addition time are emitted on demand, or into "one pulse time" operation in which the signals for an addition time are emitted in sequence, one each time a certain button is pushed. Either of these noncontinuous methods of operation permit the operator, without disturbing the flip-flop memory, to stop the ENIAC at some point and examine the neons.

The counters of the ENIAC, in general, consist of a number of flip-flops arranged in sequence and so interconnected that the following characteristics result: (1) at any given time, one, and only one, flip-flop can be in the abnormal state: (2) the reception of a pulse at the input to the counter causes the flip-flop which is in the abnormal state to be reset and causes its successor to be set: and (3) the counter can be cleared so that a specific flip-flop comes up in the abnormal state and all others in the normal state. 
Each flip-flop of a counter is called a stage and the reception of a pulse at a counter is said to advance the counter to the next stage. All counters in the ENIAC are ring counters, i.e., the first and last stages are so connected that if the counter is in its last stage and a pulse is received, the last stage is reset and the first stage is flipped into the abnormal state.

In accumulators, a 10-stage (decade) ring counter is used for each place of a 10-place number. Each stage of a decade counter corresponds to one of the digits between 0 and 9 inclusive. The sign of a number is handled by means of a PM counter which differs somewhat from the other ENIAC counters. The PM counter has 2 tubes, one for sign $\mathrm{P}$ (for positive numbers) and one for sign $M$ (for negative numbers). Here each tube is referred to as a stage. The tubes are so connected that one, and only one, of them can be conducting at a given time. While the PM counter uses two tubes as does a flip-flop, it differs from an ordinary flip-flop in that it has but one input. The PM counter is also a ring counter. The 11 counters of an accumulator are connected by carry-over circuits so that when a counter advances from 9 back to 0 , provision is made for the next counter at the left also to advance one stage.

Numbers are transmitted in the ENIAC in pulse form with the pulses for all ten decimal places and sign being given out at the same time. Trunk lines consisting of 11 conductors are used for the transmission of these pulses from one unit to another. To represent the digit $d(0 \leqslant d \leqslant 9)$ in a given place of a number, $d$ pulses are transmitted over the conductor corresponding to that place. No sign pulses are used for positive numbers; nine sign pulses, for negative numbers.

An accumulator adds a number to one it already contains by counting the pulses it receives. Since the counters in an accumulator cycle in but one direction, subtraction is treated as a form of addition through the use of a system of complements.

Let us focus attention on the 10 decade counters of an accumulator. Any integer between 1 and $10^{10}-1$ inclusive, is uniquely representable on the counters, but the numbers 0 and $10^{10}$ are indistinguishable. Hence the sum $\left(10^{10}-N\right)+N$ appears as 0 on the decade counters. The number $10^{10}-N$ is referred to as the complement of $N$ with respect to $10^{10}$.

The PM counter to the left of the decade counters permits a unique representation both of positive numbers $N$ (for $0 \leqslant N \leqslant 10^{10}-1$ ) and their complements. The PM counter can receive as its input either sign pulses (none for a positive number; nine for a negative number) or a carryover pulse from the extreme left-hand decade. The reception of an even number of pulses leaves this counter in its original state; the reception of an odd number of pulses cycles the counter to its other stage. It is now easy to verify that all the usual arithmetic properties of addition and subtraction are obeyed in the system described. There follow a few illustrative examples: The sum of 801 and 527 appears as

$$
\begin{array}{lllll}
\mathrm{P} & 0 & 000 & 000 & 801 \\
\mathrm{P} & 0 & 000 & 000 & 527
\end{array}
$$

P 0000001328. 
The difference 801 minus 527 as

$$
\begin{array}{lllll}
\text { P } & 0 & 000 & 000 & 801 \\
\text { M } & 9 & 999 & 999 & 473
\end{array}
$$

P 0000000 274;

and the difference 527 minus 801 as

$$
\begin{array}{lllll}
\text { P } & 0 & 000 & 000 & 527 \\
\text { M } & 9 & 999 & 999 & 199 \\
\hline \text { M } & 9 & 999 & 999 & 726 .
\end{array}
$$

Counters are used not only for arithmetic purposes, but also as a part of the programming circuits which determine when and how a given unit shall perform. Each unit whose operations consume more than one addition time has such a program counter. While the unit is operating, a CPP is allowed to reach the input to the counter and cycle it one stage per addition time. Each stage of a counter, it is to be remembered, is a flip-flop. Therefore, when a counter reaches a given stage, the polarity of the outputs of the flip-flop for that stage is reversed from normal. Use is made of this to cause circuits to function in a manner appropriate to the addition time of the operation. When the program ring has been cycled through its range, it causes the unit to cease operating.

In addition to their use in program counters, flip-flops have a role in another aspect of the ENIAC's programming memory. However, before continuing with this topic, we should like to in troduce a distinction between certain kinds of circuits in the various units. It is convenient in discussing the ENIAC to distinguish between the numerical circuits (which operate on signals representing numbers) and the programming circuits (which recognize when and how a unit is to operate and which then stimulate the numerical circuits to operate). In the case of most units, it is desirable to subdivide the programming circuit classification into program controls and common programming circuits. All but a few of the ENIAC units have multiple program controls, any one of which is capable of recognizing that a unit is to operate in a given way. All the program controls on a unit operate a common set of programming circuits which stimulate the numerical circuits to function in the desired manner.

Each program control consists of a set of program switches, a flip-flop, an input terminal for the reception of a program pulse, and associated tube circuits. Most program controls also have an output terminal for the transmission of a program output pulse when an operation is completed. These controls are referred to as transceiver program controls; the others, as receiver program controls. The outputs of the flip-flop in a program control are taken, ultimately, to the program switches. The points of these switches, in turn, are connected to various elements of the common programming circuits. When the flip-flops of all the program controls on a unit are in the normal state, the polarity of the signals delivered to the common programming circuits is such as to leave them quiescent. 
When, however, a program control is stimulated by the reception of a program input pulse, the polarity of its flip-flop outputs is reversed, and, these signals, routed through the program switches, activate appropriate ones of the common programming circuits. Receiver program controls are used only in conjunction with operations that take but one addition time. One of the outputs of a receiver program control allows a CPP to reach the reset input and restore the flip-flop to its normal state one addition time after it is set. Transceiver controls operate in conjunction with a program counter. A signal from such a counter allows a CPP to reset the control after the required number of addition times have elapsed. When a transceiver program control is reset, it causes a CPP to be emitted as a program output pulse.

5. Numerical Units of the ENIAC. In this section we shall consider the units which perform numerical operations: addition, subtraction, multiplication, division and square rooting, and obtaining a number from the machine's memory. For each unit, we shall state the number of program controls in order to present some measure of the machine's capacity.

Each of the 20 accumulators is capable of storing a signed 10-digit number and, as described in section 4, of receiving a number and adding it to one it already contains. The accumulator has 5 input channels. For each input channel there is a digit input terminal consisting of 11 wires and a ground. The purpose of the multiple input channels will be treated in section 6. The accumulators also have two output circuits, with a digit output terminal for each one located on the front face. Through one output (the $A$ output), the accumulator can transmit the number it stores; through the other (the $\mathbf{S}$ output), the complement of the number it stores. In any given addition time, the accumulator can either receive over one of its input channels or transmit through either or both of its outputs. The machine can be set up, as will be shown in section 6 , so that any accumulator can communicate with any other, with the function tables, and with the constant transmitter. Communication to and from the multiplier and divider is possible only through specific accumulators.

In addition to the $A$ and $S$ outputs, the accumulators have a set of "static outputs" which provide a circuit directly from each stage of a counter. The static outputs of two specific accumulators which serve as argument accumulators are delivered to the multiplier. Static outputs for sign indication are delivered from another pair of accumulators to the divider and square rooter, and finally, the static outputs of a total of 80 decade counters and 16 PM counters are connected to the printer. Numbers which are to be printed are either formed in or sent to the accumulators connected to the printer before printing is due to take place. Any numbers stored in these accumulators are always available to the printer unit; the printer, however, takes cognizance of the numbers and causes them to be punched only when instructed to do so.

Each accumulator has 8 transceiver program controls and 4 receiver controls. These controls allow an accumulator to receive or transmit and to clear or not clear after transmission. A repeat switch included in the transceiver controls makes it possible to receive or transmit up to nine times in succession. 
Each accumulator has a significant figure switch which enables the accumulator either to clear to 0 in all decades or to clear to 0 in all decades but one, selected by the switch setting, and to clear to 5 in that one. This, combined with the ability to delete the digit pulses in any desired place of a number (see section 6), makes it possible to round a number off in an accumulator. When it is desired, two accumulators can be interconnected in a special way to provide an accumulator with 20 decade counters and a PM counter and with 24 program controls.

The multiplier's numerical circuits consist essentially of those for storing products corresponding to one-digit arguments, for selecting the products appropriate to particular arguments and for emitting these products. This unit operates in conjunction with 4 accumulators (or possibly 6 if products with 10 to 20 digits are desired). Two accumulators, whose static outputs are delivered to the circuits for selecting products, hold the arguments. The multiplier unit produces the product of the entire multiplicand by one digit of the multiplier per addition time. Two multiplication tables are used, one for storing the tens digits of the fundamental products and one for storing the units digits of the products (for the product $4 \times 7$, for example, the tens digit is two and the units digit 8). The tens and units digits of the partial products are emitted simultaneously through two separate channels and accumulated separately in two accumulators. As multiplication by the digits of the multiplier proceeds, the multiplier shifts the partial products into the proper places before emitting them. When the digits of the multiplier are exhausted, the tens and units components of the products are added together in one of the 2 partial product accumulators.

The multiplier has 24 transceiver program controls. Each of these can be used to specify through which input channels argument accumulators shall receive their arguments for a given multiplication, whether the arguments are to be cleared after multiplication, how the product shall be disposed of from the final product accumulator, how many digits of the multiplier are to be used, and how many significant figures the product shall have.

The divider and square rooter operates in conjunction with 4 accumulators: one for storing the numerator (or radicand), one for the denominator (or twice-the-square-root), one for the quotient, and one for shifting (the necessity for this will be seen below). Division is performed by a method of successive subtractions or additions of the denominator to the numerator's remainder until an overdraft occurs. At that time the procedure is interrupted, the remainder from the numerator is sent to the shift accumulator for shifting one place to the left and then returned to the numerator accumulator. The divider emits components of the answer to the quotient accumulator, sending out +1 or -1 in a given place according as the denominator is subtracted from, or added to, the remainder. Square rooting is carried out by a method of successive subtractions or additions of odd numbers similar to that frequently used on desk model computing machines.

This unit has 8 transceiver program controls. Each control includes a program switch for specifying either division or square rooting anci other program switches offering options similar to those offered by the multiplier program controls.

Both the multiplier and the divider and square rooter are semi-permanently connected to their associated accumulators for the communication 
of numerical and programming signals involved in these operations. When these units are not in use, their associated accumulators are free to be employed in the same way as any others.

Each of the three function tables can store 104 entries consisting of 12 digits and 2 signs each. On its front face, the function table has a digit input terminal for receiving an argument and 2 digit output terminals. A sign, and 6 of the 12 digits, are emitted through each of these output terminals. By means of special adaptors (see section 6) the outputs of both terminals may be combined so that entries for one or more functions may be derived from each function table.

Each of 11 transceiver program controls on the function table permits the operator to specify transmission of the functional value corresponding to a particular value of the argument or to any of 4 neighboring values of the argument, or to specify transmission of the negative of the preceding. Multiplication by small constants is also possible along with the transmission since each control includes a repeat switch for specifying transmission up to 9 times in succession. The options afforded by the function table's program controls make it easy to set up the machine for interpolation routines.

The constant transmitter has 30 transceiver program controls. Twentyfour of these give access to the total of 16 signs and 80 digits which the reader reads from a punched card. These 24 controls are arranged in groups of 6 which operate common programming circuits for selecting any signed 5- or 10-digit group out of a total of 4 signs and 20 digits. The remaining 6 program controls are used for the 4 signs and 20 digits which can be set up on the constant transmitter's switches.

6. Interconnections for a Particular Computation. Before the ENIAC can carry out a computation, not only must the program switches be set, but also the various units of the ENIAC must be connected for the communication of numerical and programming signals in such a way as to meet the needs of the particular computation. Multiple trunk lines running past the units are provided for this purpose. Both the digit and program trunk lines consist of 11 conductors each, mounted on trays. The digit trunks have 12-point terminals spaced so that there is one in front of each panel. These terminals are connected to digit terminals on the units by digit cables. The program trunks have a group of 11 separate 2-point terminals instead of the 12-point digit terminals. Each of these terminals can be connected to a program pulse input or output terminal by means of a program cable. Within limits, any number of digit terminals can be plugged into the same digit trunk or any number of program terminals into the same line of a program trunk. The program trunks are stacked below the panels containing the program switches and the digit trunks above the switch panels (see Fig. 1).

Adaptors, consisting of specially wired plug and socket assemblies, are also used in the transmission of digits. Each conductor of a digit tray ordinarily carries the pulses for a specific place of a 10-place number or for sign indication. Adaptors are used when it is desired to effect a special relationship between the digit places of the transmitting and receiving unit. Shifter adaptors are used to effect multiplication by powers of 10 . Deleter adaptors 
open certain circuit lines so as to eliminate the pulses for one or more places of the number.

The five separate digit input channels on an accumulator make it possible for an accumulator to receive from any one of $\mathbf{5}$ different digit trunks. This makes it possible for numerical operations to be carried on in different parts of the machine simultaneously. The different input channels also are useful in that they enable reception through different adaptors at various times.

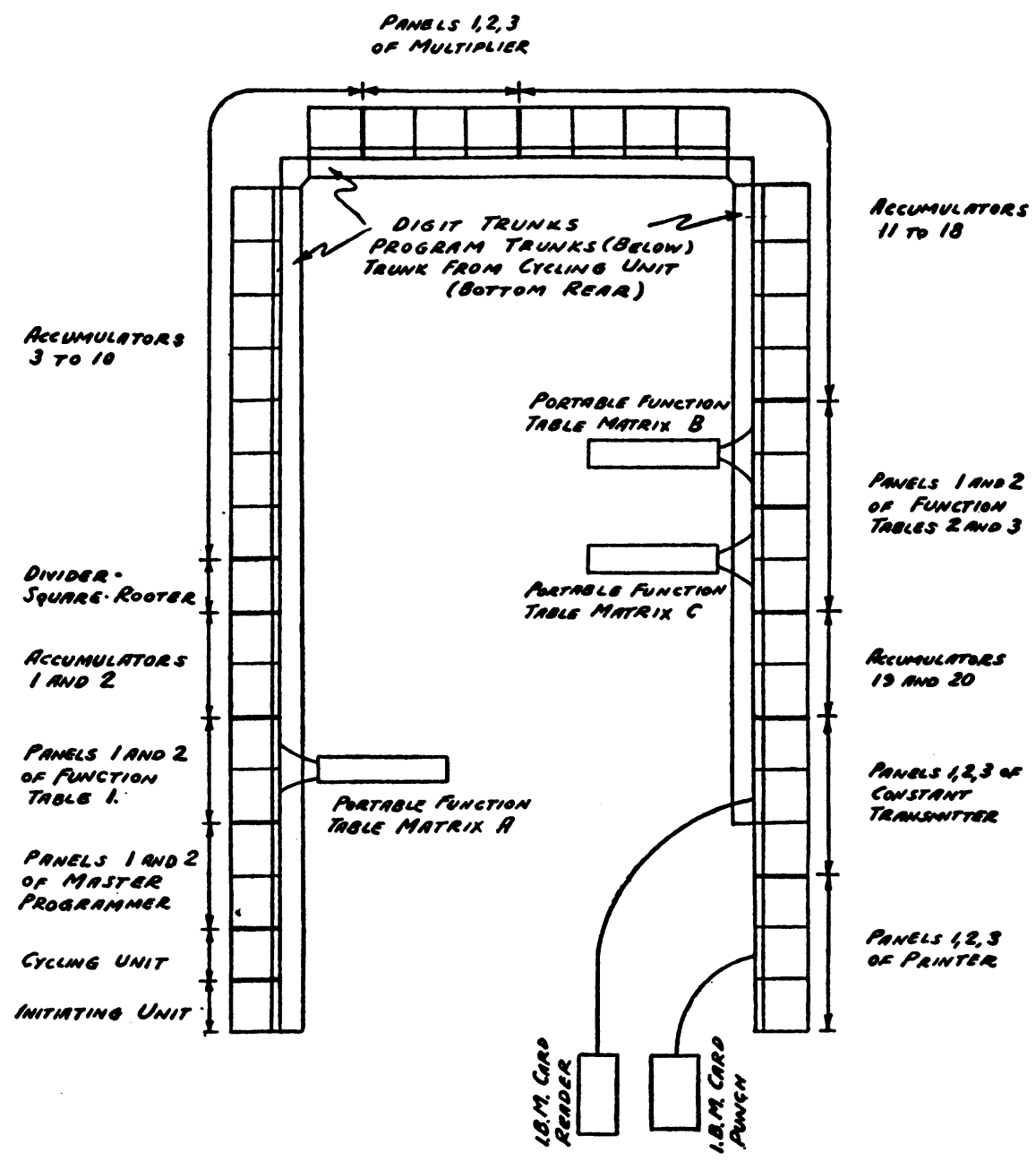

FIG. 2.

7. Sequencing and the Master Programmer. We shall consider in this section the procedure for carrying out a computation by means of this machine. For the ENIAC, as for human computers, the computation must consist of a series of arithmetical operations. Thus, for example, if the computation involves evaluating integrals, numerical integration formulae must be introduced. Another preliminary step before the computation can 
be given to the machine consists of planning for the storage of numbers required in the computation by means of the function tables, the accumulators, and possibly, punched cards. Incidental to this is the consideration of establishing digit connections between the various units so as to make possible the required transfers of numbers from one part of the machine to another.

We next consider how to instruct the ENIAC in its routine for a particular computation. One or more program controls must be devoted to each arithmetical operation of the computation. For example, if accumulator 1 stores the number $a$ and accumulator 2 the number $b$, then, to form $a-b$, accumulator 2 must be instructed to transmit through its $S$ output, and accumulator 1 must be instructed to receive over a channel connected to the $S$ output of accumulator 2 .

To tie the individual operations into a sequence in which one operation or a group of operations is initiated upon the completion of another, use is made of the transceiver program controls. Recall that when a transceiver control completes an operation, it emits a program output pulse. If the program output pulse terminal of such a control is connected to a line in a program trunk, all program controls whose input terminals are connected to this line are stimulated when the transceiver control completes its operation. To illustrate the method of sequencing the ENIAC, let us consider the trivial computation for simultaneously generating a table of squares and cubes. We assume that $n, n^{2}$, and $n^{3}$ are stored in certain accumulators and proceed inductively to form $n+1,(n+1)^{2}$, and $(n+1)^{8}$. The steps for these computations are shown schematically in Fig. 3.

The questions that remain to be considered for this computation are: (i) How is the computation initiated?; (ii) How could this sequence be repeated $r$ times?; and (iii) How could a different sequence be initiated after the $r$ th repetition? The first matter is taken care of by means of a program control on the initiating unit which, under the control of a push button, causes an initiating pulse to be emitted. By delivering the initiating pulse to program line A-1, we could provide for starting the computation. The problems of iterating the sequence into a chain and linking another sequence or chain to it are provided for by the master programmer.

The master programmer consists of ten units, each of which is capable of counting program pulses and of switching program connections. Each unit has a 6-stage counter, called a stepper counter and may háve from 1 to 5 associated decade counters which are connected to one another for carryover purposes. For each stepper-decade combination, there is one input terminal and there are 6 output terminals. When a pulse is received at the stepper, it is registered in the decades and a pulse is emitted through one of the 6 output terminals. The stage of the stepper counter determines through which output terminal the pulse is emitted.

Control of the stage of the stepper counter is dependent upon the following considerations: Associated with each stage of a stepper counter is a group of so-called decade switches. Let us say that the setting of the decade switches associated with stage $s$ of a stepper counter is $d_{8}\left(0 \leqslant d_{8}<10^{5}\right)$. Then, if the stepper counter is in stage $s$, it emits a pulse from the output terminal associated with stage $s$ on each of $d$, occasions. Input pulse number $d_{\text {, }}$ moreover, causes the stepper counter to advance to stage $s+1$ and causes 

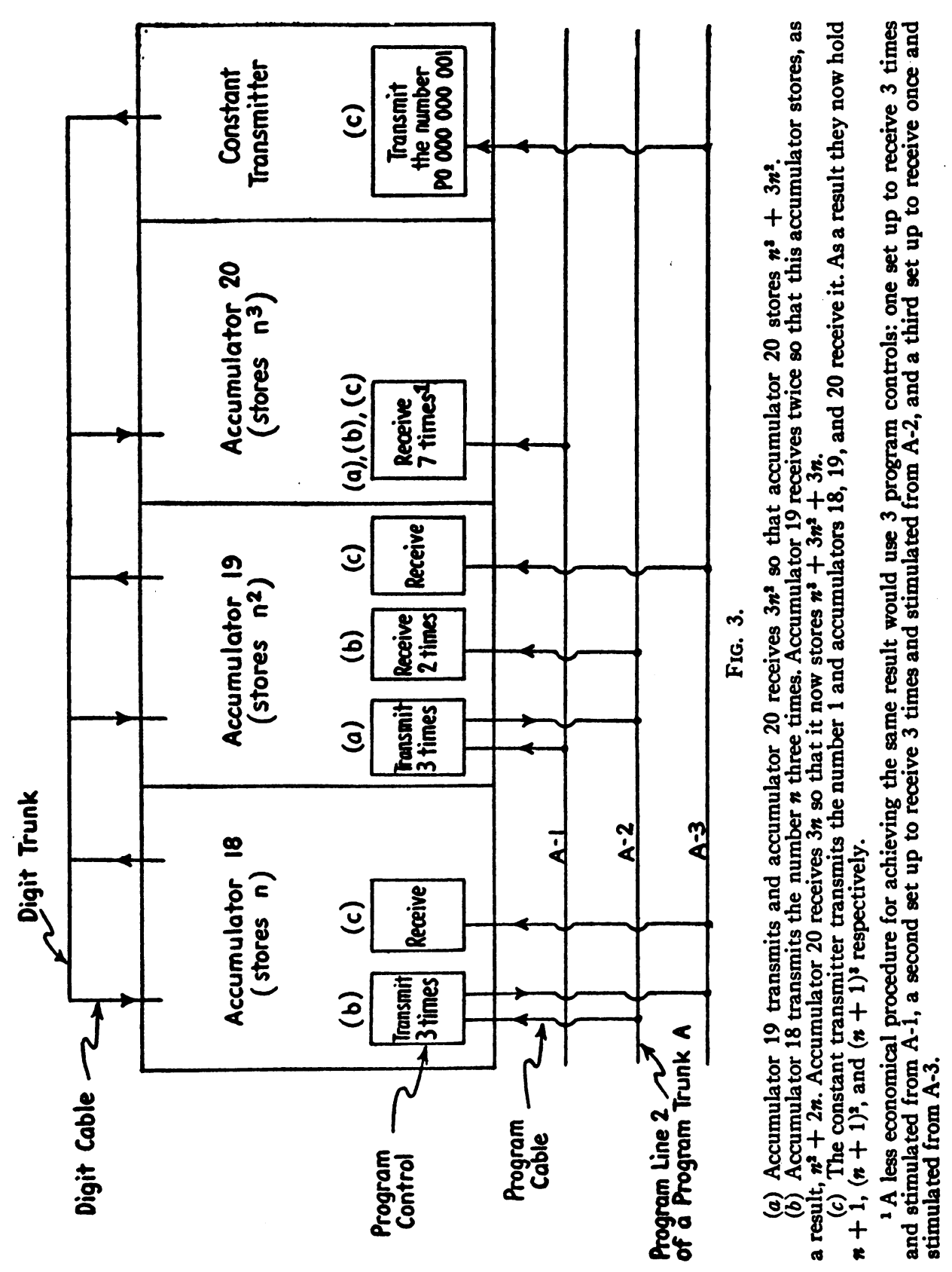


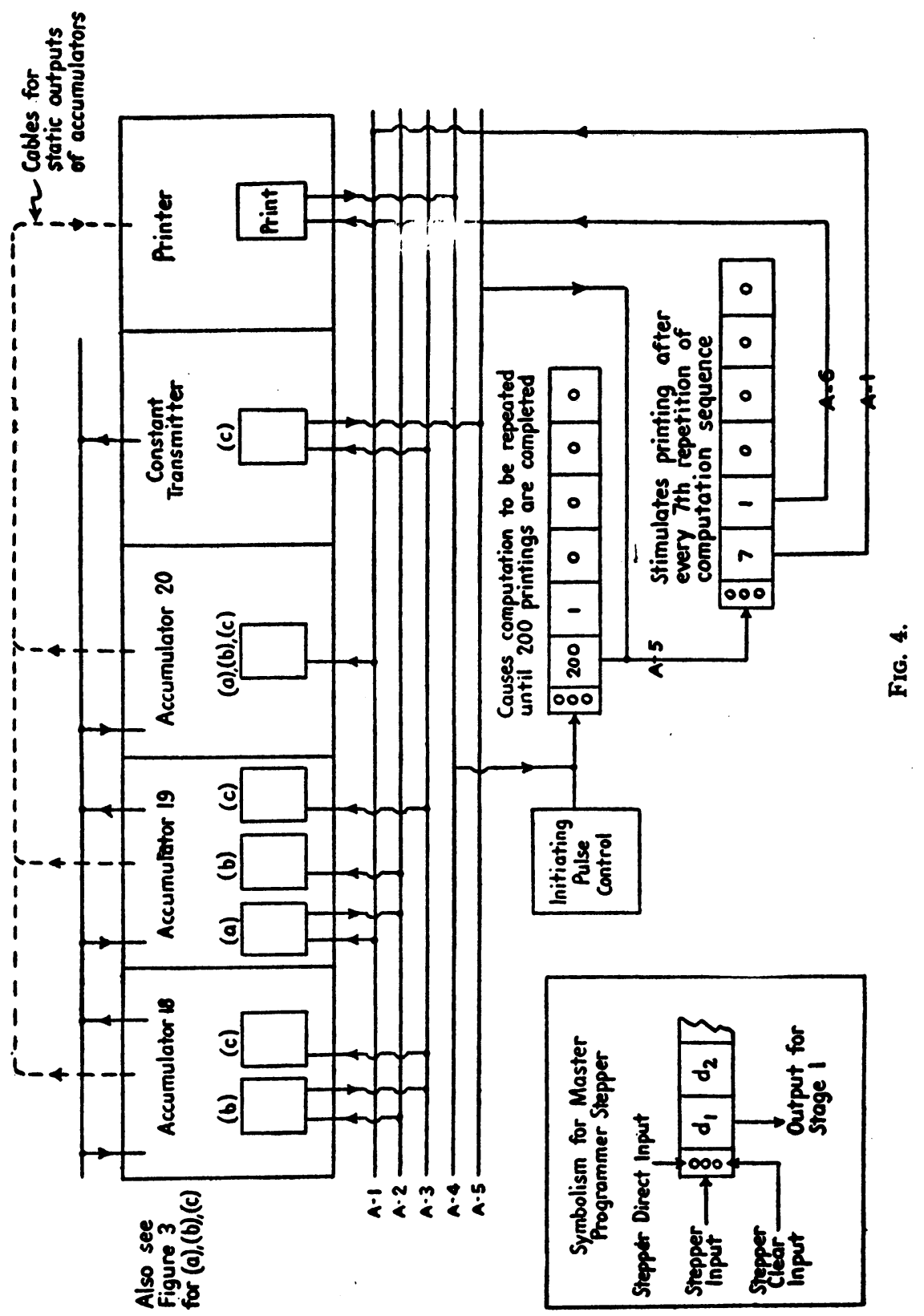


the associated decade counters to clear to 0 . Besides the preceding, each stepper counter has a direct input which allows the stepper counter to be stepped independently of the decade counters and a clear input which allows the stepper counter to be cleared back to its first stage from any stage. It might be remarked that digit pulses, as well as program pulses, may be brought to the stepper direct input.

We will now return to the problem discussed above to illustrate how the master programmer may be used to link program sequences. Let us suppose that it is desired to repeat the computations described 7 times and then to print. Suppose, furthermore, that computations are to cease after 200 printings A complete plan for this computatic. is given in Fig. 4.

In the ENIAC, programs may be linked together either serially, as described above, or the choice of routine may be made to depend on the magnitude of a number. The criterion may be the magnitude of the digit in a specific place of a number or it may be the sign of a number. Either form of magnitude discrimination can be accomplished by sending pulses from some lead of a digit output terminal to a stepper direct input.

The ENIAC has, of course, been designed to handle far more complicated and tedious computations than the one discussed above which required the use of less than 4 percent of the machine's programming capacity. This illustrative problem is, however, useful for indicating how the machine can be instructed in its routine for a particular computation.

Institute for Advanced Studies

H. H. Goldstine \& Adele Goldstine

\section{Scientific Computing in Great Britain}

1. Introduction: Commercial Machines in Scientific Computing. The commercial calculating machine has been used in scientific work, after a fashion, for many years. Twenty years ago one or two hand-operated machines were to be found in the mathematics departments of British universities, used by graduate students. But a great stimulus to their better and wider use was given by $\mathrm{L}$. J. COMRIE in lectures given at the London School of Economics in $1926^{1}$ and $1927,{ }^{2}$ in which he stressed the importance of the intelligent and resourceful use of commercial machines.

About the same time Comrie described the "end-figure" method" of constructing mathematical tables by subtabulation, and a means of using this method on Hollerith punched-card equipment. Four years later 4 came his description of the Burroughs Class 11 machine and its use for integration from second differences. About the same time he described his application of the Hollerith tabulator to E. W. BRown, Tables of the Moon, which still stands as a remarkable achievement.

His next great advance was the use of the six-register National Accounting machine as a "difference-engine" to handle problems in finite differences. As a result there are now fifteen National machines in use in research establishments in Great Britain, several times as many as there are i n similar use in the United States, where the machine originated!

The story of the adaptation of commercial machines to uses in the scientific field in Great Britain is almost wholly the story of Comrie's life 